\title{
The Landscape of Clinical Implementation of Pharmacogenetic Testing in Central China: A Single-Center Study
}

\author{
Jingmin Zhang $\mathbb{D}^{1,2}$ \\ Guangzhao Qi $\mathbb{1}^{1,2}$ \\ Chao Han ${ }^{1,2}$ \\ Yubing Zhou ${ }^{1,2}$ \\ Yongjie Yang' \\ Xinru Wang ${ }^{1,2}$ \\ Suna $\mathrm{Liu}^{3}$ \\ Xiaojian Zhang ${ }^{1,2}$
}

'Department of Pharmacy, The First Affiliated Hospital of Zhengzhou University, Zhengzhou, People's Republic of China; ${ }^{2}$ Henan Key Laboratory for Precision Clinical Pharmacy, The First Affiliated Hospital of Zhengzhou University, Zhengzhou, People's Republic of China; ${ }^{3}$ Newborn Screening Center, Department of Pediatrics, The Third Affiliated Hospital of Zhengzhou University, Zhengzhou, People's Republic of China
Correspondence: Guangzhao Qi; Xiaojian Zhang

Tel +86-37l66295269

Email qiguangzhaol223@sina.com; zhangxiaojianI5@I26.com
Purpose: Pharmacogenetic testing is recognized as the major method for the individualized pharmacotherapy in clinical pharmacy practice, but information about the clinical implementation of pharmacogenetic testing in China is limited. The present study aimed to determine the situation of clinical implementation for pharmacogenetic testing in central China.

Methods: The study is conducted in the department of clinical pharmacy in The First Affiliated Hospital of Zhengzhou University, Zhengzhou, China. We collected and analyzed pharmacogenetic testing results from November 1, 2013 to November 2, 2018 in our hospital, which were checked in the electronic medical record system. The main outcome measures were the number and type of pharmacogenetic testing across five years.

Results: A total of 47,265 (56.9\% male, mean age $=51.5$ years) pharmacogenetic testing results were obtained with an average annual rate of growth of $63.0 \%$ across five years. A $50.2 \%(23,748 / 47,265)$ of all the pharmacogenetic testing results were for the determination of cytochrome P450 2C19 (CYP2C19) *2, *3 genotypes, and 41.7\% were for the methylene tetrahydrofolate reductase (MTHFR) C677T genotype. The number of departments performing the pharmacogenetic testing was 35, 63, 55, 52, 52 and 39 for 2013-2018, respectively, and the main top five departments were cardiology, psychiatry, ICU, cardiac surgery and intervention.

Conclusion: Clinical implementation of pharmacogenetic testing in China is growing rapidly, but the types and implementing departments of pharmacogenetic testing were limited. Our present study reported the real-world implementation modality of pharmacogenomic tests in China. It will help us to understand the testing of pharmacogenetics in China in order to promote the rational development of pharmacogenetics.

Keywords: clinical implementation, pharmacogenomics, pharmacogenetics, China, preemptive

\section{Introduction}

Genetic variation of drug absorption, distribution, metabolism and excretion (ADME) genes could impact the inter-individual drug response differences among patients. ${ }^{1}$ As one of the emerging approaches to precision medicine, pharmacogenomics selects drugs and doses based on a patient's genetic features. ${ }^{2}$ Decades of pharmacogenetics (PGx) study identified numerous gene-drug interactions, such as CYP2C19 (cytochrome P450 2C19) and clopidogrel, CYP2D6 (cytochrome P450 2D6) and tamoxifen, SLCO1B1 (solute carrier organic anion transporter family member $1 \mathrm{~B} 1)$ and rosuvastatin. ${ }^{3,4}$ The increasing evidence 
supports the integration of pharmacogenetic information into the clinic. Retrospective study on several large clinical trials identified that the clinical outcomes of acute myocardial infarction patients treated with clopidogrel were associated with CYP2C19 genotypes, but not in the acute coronary syndromes or atrial fibrillation patients. ${ }^{5,6}$ Prospective study on the genetically guided prescription replicated the association between genetic variants and drug responses. ${ }^{7}$ These results leaded to the initiation and acceleration of the widely used preemptive PGx testing in clinical settings.

The attitudes of patients, healthcare professionals and insurance payers towards the preemptive PGx testing were diverse, which influenced the adoption of PGx testing in clinic. ${ }^{8-10}$ Usually, patients especially with a cancer tended to obtain a promising benefit of genetic testing, whereas physicians harbored a concern for genetic testing. The different phenotype could be caused by inter-individual differences in genotype. ${ }^{11,12}$ The inconsistency between the patient's genotype and anticipated drug response observed by clinicians might impair their confidence of implementing PGx test in future. Deciphering the realworld clinical implementation of PGx test could find the gap between the pharmacogenomics research community and healthcare professionals, which provoked further study on the genetic variants and certain drug response. ${ }^{13}$

A number of resources, including the US Food and Drug Administration (FDA) and the Clinical Pharmacogenetics Implementation Consortium (CPIC) guidelines (https:// cpicpgx.org/), have been published on multiple clinical evidence and recommendations for individual gene-drug pairs. However, conventional pharmacogenetic testing has been slow to translate into clinical application. ${ }^{14}$ Several programs including The Pharmacogenomics Research Network (PGRN) Translational Pharmacogenetics Program and Ubiquitous Pharmacogenomics Consortium (U-PGx project) (www.upgx.eu) in the Netherlands had aimed to utilize preemptive PGx testing for clinical implementation. ${ }^{15}$ Considering the infrequent PGx guided prescribing in clinic under the nationwide availability of PGx guidelines in electronic medical record system, some study investigated the adoption of community pharmacist initiated PGx test in primary care. ${ }^{16}$ Despite the increased interest in genetics in the public sphere, the rate of adoption of PGx testing in the clinical setting has been uneven. Especially, the information about the clinical implementation of PGx testing in China is limited. To determine the situation of clinical implementation for pharmacogenetic testing in central China, we analysed 47,265 real-world pharmacogenetic testing results from November 1, 2013 to November 2, 2018 in our hospital, which to the best of our knowledge might be the first study to date on the clinical implementation of PGx testing in China.

\section{Materials and Methods}

\section{Setting Description}

The First Affiliated Hospital of Zhengzhou University in central China is an 8000-bed-Grade IIIA Provincial Ministry Hospital. The hospital has 20 national key clinical specialty construction projects, and 3 national regional medical centers. The genetic testing platform relies on the department of pharmacy of our hospital and Henan Key Laboratory for Precision Clinical Pharmacy. Therapeutic individualization has always been an important objective for our department.

Clinical implementation of gene-drug(s) pairs includingCYP2C9 and VKORC1- warfarin, ${ }^{17}$ CYP2C19clopidogrel, ${ }^{18}$ CYP2C19-proton pump inhibitors, ${ }^{19}$ CYP2C19voriconazole $^{20}$ and CYP2D6/CYP2C19-selective serotonin reuptake inhibitors, ${ }^{21}$ etc., are recommended in the drug specifications and are referenced in the CPIC guideline. In addition, methylenetetrahydrofolate reductase $(M T H F R)$ is an enzyme involved in the folate metabolic pathway with two polymorphic variants: C677T and A1298C. Several metaanalyses have reported a possible association between MTHFR C677T gene polymorphisms and infertility and unexplained recurrent pregnancy loss in Asian populations, whereas this association was not evident in Caucasian populations. ${ }^{22-25}$ Moreover, increasing evidence demonstrated that MTHFR C677T and A1298C is associated with psychiatric diseases. ${ }^{26}$ In neurological and psychiatric disorders, the MTHFR C677T polymorphism was reported to be associated with Alzheimer's disease, schizophrenia and bipolar disorder in Asian populations, but not in Caucasian populations. ${ }^{27,28}$ Therefore, determining MTHFR genotype in psychiatric patients who are responsive or non-responsive to conventional medication is important for personalized therapeutic management of psychiatric disorders. $^{26}$

Multiple phenotype categories have been identified, namely, "poor metabolizer", "intermediate metabolizer", "extensive (normal) metabolizer", and "ultrarapid metabolizer". Based on the test results, the pharmacist alerts the clinician to adjust the dose administered or to be aware of adverse drug reactions and drug interactions (Table S1). Also, women with TT genotype may be given a higher dose or longer duration of pre-pregnancy supplementation, 
depending on individual circumstances (Class I recommendation, Level B evidence), ${ }^{29}$ and blood homocysteine levels need to be monitored for others.

\section{Data Resources and Analysis}

The data of PGx testing were collected and checked in the PACS (picture archiving and communication system), including imaging testing such as CT (computed tomography), MRI (magnetic resonance imaging) and pathology results from November 1, 2013 to November 2, 2018 in The First Affiliated Hospital of Zhengzhou University, Zhengzhou, China. Each PGx testing item includes the name, sex, and age of the tested patient, outpatient or inpatient, patient ID, PGx ID, department and the date and time of the testing. The testing data were shown in supporting information (Table S1). As shown in Table S1, the multidisciplinary team, including physicians, nurses, genetic counsellors, and pharmacists will need to combine their expertise to provide optimal pharmacogenomic therapy for patients in our hospital. We recognized the different PGx testing of one same patient as isolated testing. The different divisions of the same department were calculated as the same department. The testing obtaining a different PGx ID was recognized as one isolated PGx testing, and ultimately a total of 47,265 PGx testing results were obtained.

We used descriptive statistics to characterize demographic and clinical characteristics of patients receiving PGx tests. Specifically, counts and percentages described categorical variables, while means, medians, and ranges described continuous variables. The average annual growth rate of PGx testing was calculated as $r=\sqrt[5]{\frac{n 2018}{n 2013}}-1$, of which $r$ is the average annual growth rate, n2018 and n2013 are the number of PGx testing for 2018 and 2013, respectively. The age distribution of 47,265 PGx testing was demonstrated as histogram and kernel density estimation created by the "base" package in the software of R (https://www.r-project.org/). The pie charts of departments performing the PGx testing each year were created in Excel 2020 (Microsoft Corporation, WA, USA). The study was approved by the Clinical Research Ethics Committee of the First Affiliated Hospital of Zhengzhou University and conducted in accordance with the Declaration of Helsinki. Written informed consents were obtained from all patients.

\section{Results}

\section{The Clinical Implementation of PGx Testing in China Grown Rapidly}

The number of PGx testing grown from 991 in 2013 to 11,393 in 2018 with an average annual growth rate of $63.0 \%$ across five years (Table 1). A $95.3 \%(45,058 / 47,265)$ of the PGx testing were performed for inpatient, while only $4.7 \%(2,207 /$ 47,265) for outpatient. Of the 2207 outpatient PGx testing, $90.2 \%$ were performed for female patient, and $87.4 \%$ for the genotyping of MTHFR C677T. The most department performing outpatient PGx testing was obstetrics clinic (74.4\%) and the average age of the patients for the 2207 outpatient PGx testing was 30.6 years (range from 0 to 83 years). However, in total, $56.9 \%$ of the 47,265 PGx testing were performed for male patient (Table 1) and the average age of the patients for the 47,265 PGx testing was 51.5 years (range from 0 to 109 years). Furthermore, the kernel density of age distribution for 47,265 PGx testing showed four peaks (Figure 1A and B), which might suggest the onset age for psychiatry (the first two peaks) and cardiovascular disease (the last two peaks).

\section{The Majority of PGx Testing Type Were Limited to CYP2C19 and MTHFR}

Over half of the 47,265 PGx testing $(50.2 \%, 23,748 / 47,265)$ were performed for the genotyping of $C Y P 2 C 19 * 2, * 3$, and $41.7 \%$ of the 47,265 PGx testing $(19,733 / 47,265)$ were for the genotyping of MTHFR C677T. The percentage of PGx testing for warfarin (CYP2C9 (cytochrome P450 2C9) *2, *3 and VKORC1 (vitamin K epoxide reductase complex subunit 1) G-1639A) and $A L D H$ (aldehyde dehydrogenase) Glu504Lys were $7.1 \%$ and $0.9 \%$, respectively. The number of PGx testing each year from 2013 to 2018 were also in the order of CYP2C19 $>$ MTHFR $>$ CYP2C9 and VKORC1 > ALDH. The departments performing CYP2C19 genetic testing are mainly concentrated in the cardiology $(52.1 \%)$ and intervention department (15.0\%). $70.4 \%$ of the genotyping of CYP2C19 were for the dose adjustment of the drug clopidogrel and $15.9 \%$ for omeprazole, $3.6 \%$ for sertraline, $3.1 \%$ for pantoprazole and other drugs including valproic acid, voriconazole, escitalopram, cyclophosphamide, lansoprazole, fluoxetine, clomipramine, esomeprazole, diazepam, phenobarbital, and rifampicin (totally 7\%). The main departments for MTHFR, (CYP2C9 and VKORCl) and ALDH at 5 years were psychiatry $(42.5 \%)$, cardiac surgery $(46.2 \%)$ and gastroenterology $(69.3 \%)$ respectively.

\section{Few Departments Performing the Majority of PGx Testing}

The number of departments performing the pharmacogenetic testing was 35, 63, 55, 52, 52 and 39 for 2013-2018, respectively (Table 1). After the initiation of PGx testing in November 1, 2013, the number of departments 
Table I Characterization of 47,265 PGx Testing

\begin{tabular}{|l|l|l|l|l|l|l|l|l|l|l|}
\hline \multicolumn{2}{|c|}{ Number of PGx Testing } \\
\hline Year & Outpatient & Inpatient & Male & Female & $\begin{array}{l}\text { CYP2C19 } \\
(* 2, * 3)\end{array}$ & $\begin{array}{l}\text { MTHFR } \\
\text { C677T }\end{array}$ & $\begin{array}{l}\text { CYP2C9 (*2, *3) } \\
\text { and VKORCI } \\
\text { (G-1639A) }\end{array}$ & $\begin{array}{l}\text { ALDH } \\
\text { Glu504Lys }\end{array}$ & PGx & Department \\
\hline 2013 & 11 & 980 & 587 & 404 & 720 & 165 & 105 & 1 & 991 & 35 \\
\hline 2014 & 304 & 6873 & 4167 & 3010 & 3527 & 2976 & 592 & 82 & 7177 & 63 \\
\hline 2015 & 349 & 7977 & 4735 & 3591 & 3730 & 3690 & 826 & 80 & 8326 & 55 \\
\hline 2016 & 296 & 8823 & 5381 & 3738 & 4603 & 3822 & 630 & 64 & 9119 & 52 \\
\hline 2017 & 528 & 9731 & 5767 & 4492 & 5377 & 4217 & 563 & 102 & 10,259 & 52 \\
\hline 2018 & 719 & 10,674 & 6268 & 5125 & 5791 & 4863 & 638 & 101 & 11,393 & 39 \\
\hline $2013-2018$ & 2207 & 45,058 & 26,905 & 20,360 & 23,748 & 19,733 & 3354 & 430 & 47,265 & 84 \\
\hline
\end{tabular}

Notes: CYP2C19 *2, NG_008384.3:g.24179G>A, dbSNP:rs4244285; CYP2CI9 *3, NG_008384.3:g.22973G>A, p.W212X, dbSNP:rs4986893; CYP2C9 *2, NG_008385.2: g.9133C>T, p.RI44C, dbSNP:rs 1799853; CYP2C9 *3, NG_008385.2:g.48I39A>C, p.1359L, dbSNP:rs 1057910.

Abbreviations: PGx, pharmacogenetics; CYP2C19, cytochrome P450 2C19; MTHFR, methylene tetrahydrofolate reductase; CYP2C9, cytochrome P450 2C9; VKORCI, vitamin $\mathrm{K}$ epoxide reductase complex subunit I; $A L D H$, aldehyde dehydrogenase.

performing the PGx testing reached its maximum of 63 in 2014, but subsequently decreased in the following years and only 39 departments performed PGx testing in 2018. The department performing the most of 47,265 PGx testing was cardiology $(26.3 \%$, Figure 2, ) and the percentage for psychiatry, intervention, ICU (intensive care unit and emergency ICU) and cardiac surgery and was $18.4 \%$, $11.8 \%, 10.7 \%$ and $9.5 \%$, respectively. The top five departments performed the majority of 47,265 PGx testing

A

Kernel Density of Age Distribution for 47,265 PGx testing

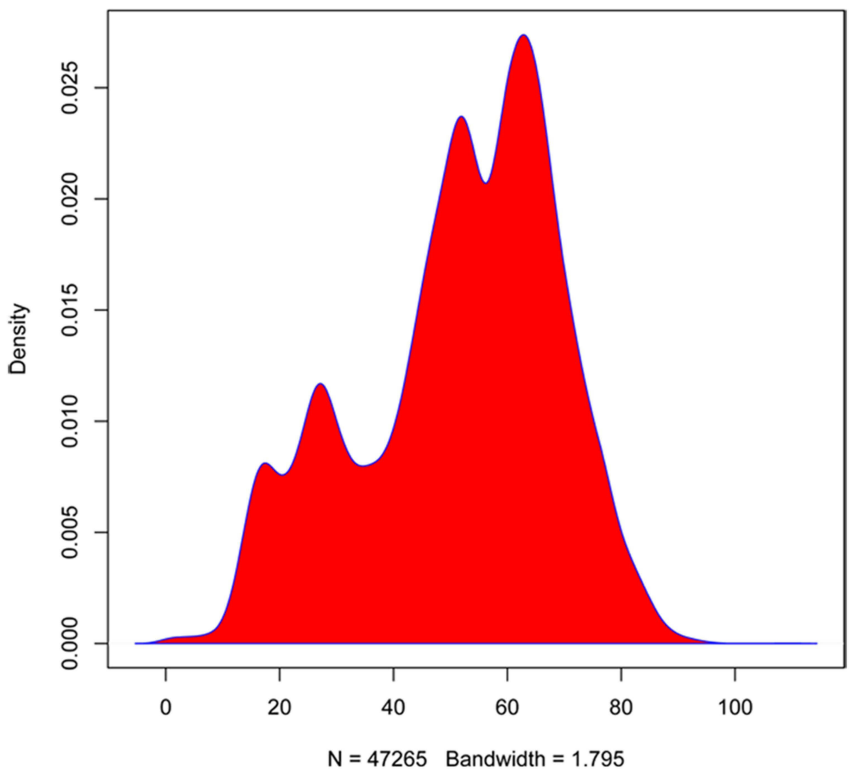

(76.7\%). What is more, for each year from 2013 to 2018, the top five departments also performed over $75 \%$ of the total annual PGx testing (Figure 3A-F). In addition, in order to further understand the detection rate of PGx in the inpatient departments, we summarized the conditions of inpatients and tested patients in the top five departments, as shown in Table 2, psychiatry had a higher detection rate than any other department every year except 2013, which was closely related to the use of psychotropic drugs.

\section{B Histogram of age distribution for 47,265 PGx testing}

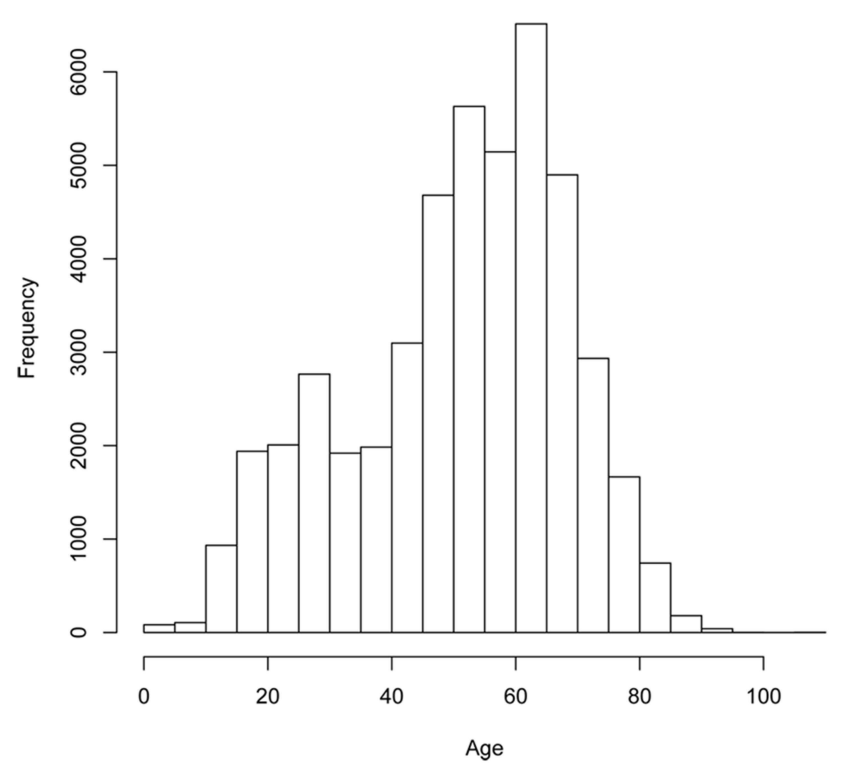

Figure I Age distribution of 47,265 pharmacogenetic testing results. Age distribution in (A) kernel density estimation and (B) histogram. 


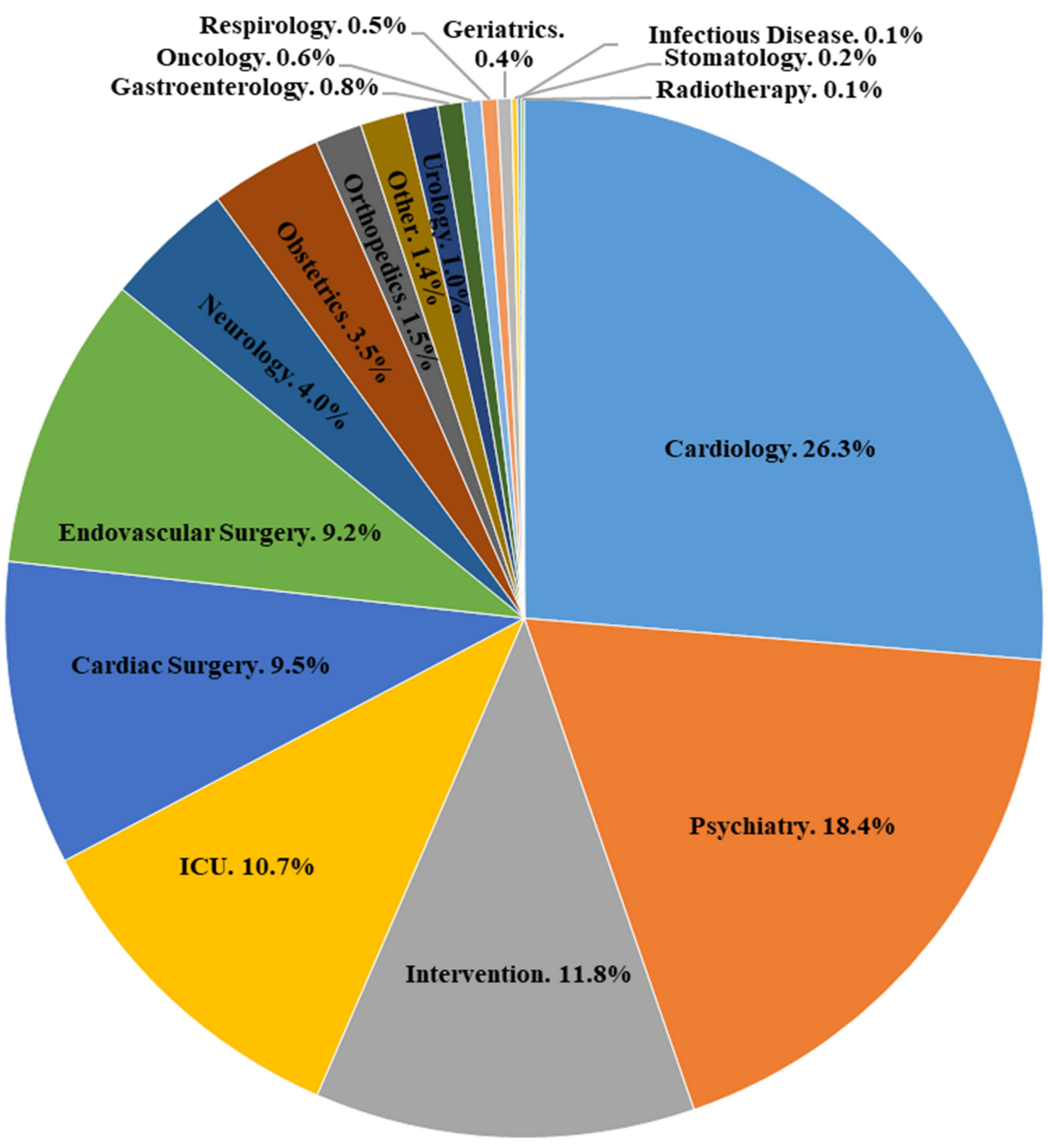

Figure 2 Departments distribution of 47,265 pharmacogenetic testing from 2013 to 2018 .

\section{Discussion}

Clinical implementation of pharmacogenomics improves the safety, efficacy, and cost-effectiveness of drug treatments. Decades of pharmacogenetics and pharmacogenomics studies worldwide accumulated a large number of data and strong evidence demonstrating the association of genetic variants with inter-individual differences for drug response. Barriers to the clinical application of PGx include short of evidence for clinical application, lack of clinical guidelines and translation of whole-genome sequencing results into PGx recommendations. However, with the clinical application of genotyping technologies, these obstacles are being addressed or overcome. ${ }^{13}$ Our present study analyzed a total of 47,265 pharmacogenetic testing results, which were obtained with an average annual rate of growth of $63.0 \%$ across five years from November 2013 to November 2018. The significant and rapid increase of PGx testing might result from mainly three reasons:
(I) full coverage of PGx tests and $100 \%$ reimbursement in the three mainstream kinds of Chinese health-care systems, ie, urban workers medical insurance, medical insurance for nonworking urban residents and the new rural cooperative medical care system; (II) annually increasing incidence of diseases such as cadiocerebrovascular disease in China; (III) awareness of addressing a disease and increased ability to pay as the economy development of China.

The knowledge of and opinions on the PGx testing among the stakeholders including health-care providers (physicians and pharmacists), health administrative departments, and patients impacted significantly the clinical implementation of PGx. Although physicians, pharmacists and patients have been enthusiastic towards PGx adoption in clinic, the main barriers including costs, ethical issue, and absence of clinical guidelines had impeded the widely carried out in primary care. ${ }^{8}$ In the present study, the majority of 47,265 PGx testing were limited 

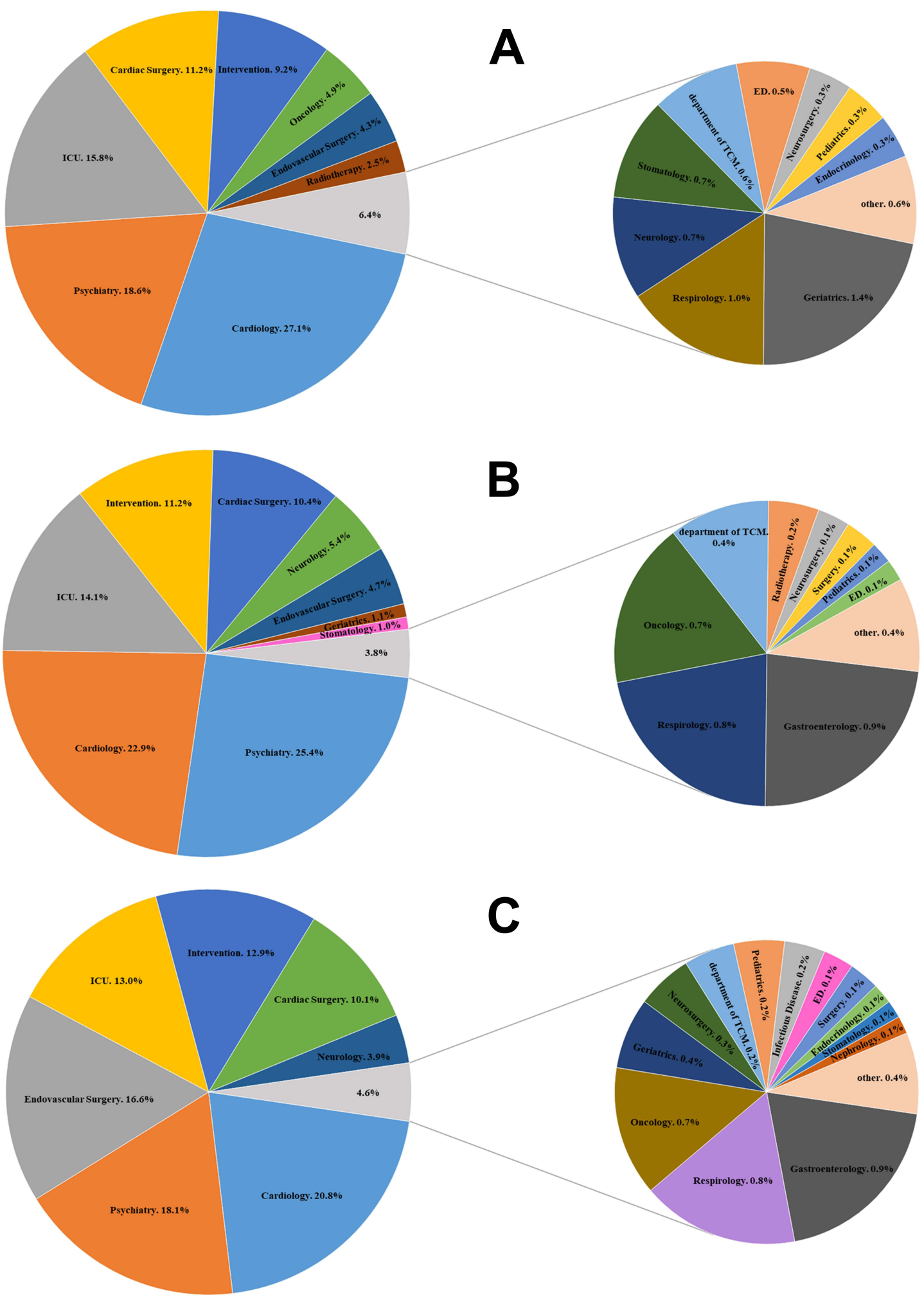

C

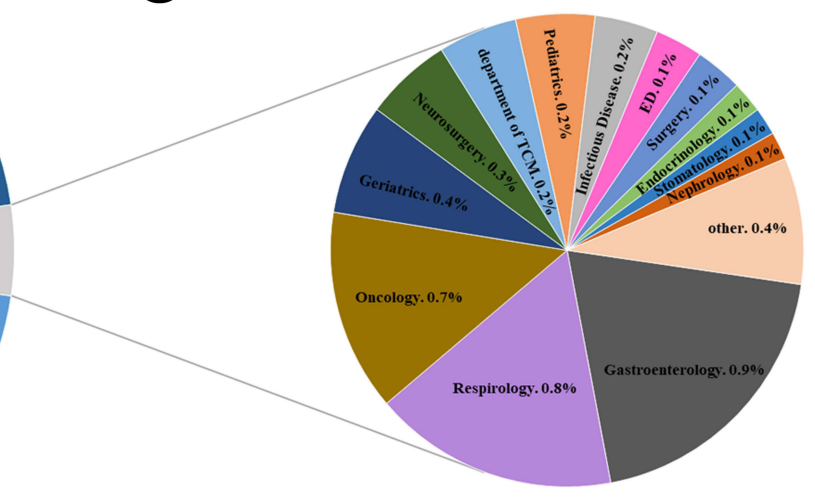

Figure 3 Continue. 

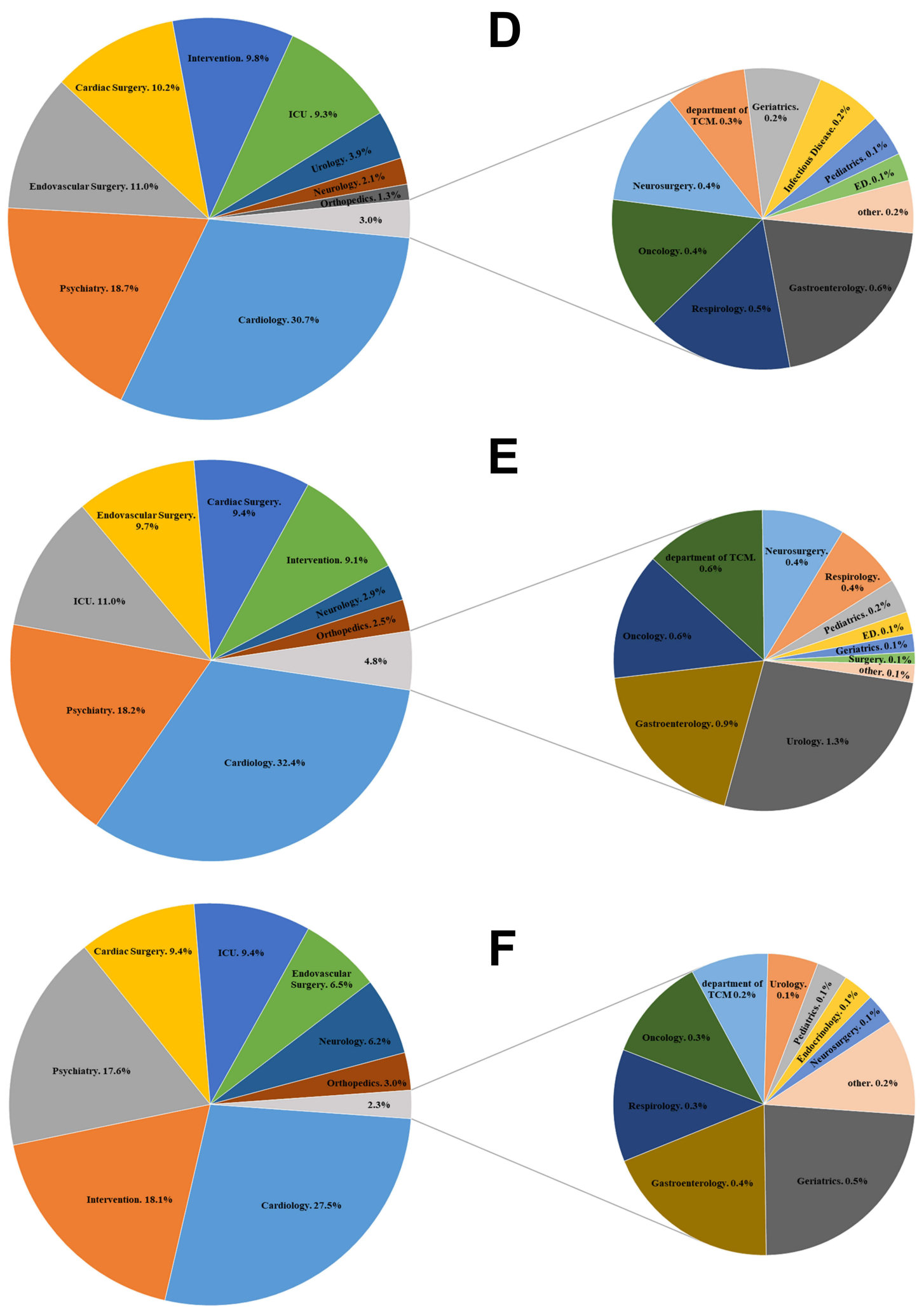

Figure 3 Departments distribution of annual pharmacogenetic testing results from 2013 to 2018. (A) 2013, (B) 2014, (C) 2015, (D) 2016, (E) 2017, (F) 2018. 
Table 2 The Detection Rate of PGx Testing in the Inpatient Departments

\begin{tabular}{|c|c|c|c|c|}
\hline Year & $\begin{array}{c}\text { Top Five } \\
\text { Departments }\end{array}$ & $\begin{array}{c}\text { Number of } \\
\text { Tested Patients }\end{array}$ & $\begin{array}{l}\text { Number of } \\
\text { Inpatients }\end{array}$ & $\begin{array}{l}\text { Rate } \\
\text { (\%) }\end{array}$ \\
\hline \multirow[t]{5}{*}{2013} & Cardiology & 266 & 2724 & 9.8 \\
\hline & Psychiatry & 183 & 608 & 30.1 \\
\hline & ICU & 155 & 193 & 80.3 \\
\hline & Cardiac Surgery & 110 & 570 & 19.3 \\
\hline & Intervention & 90 & 712 & 12.6 \\
\hline \multirow[t]{5}{*}{2014} & Psychiatry & 1753 & 3490 & 50.2 \\
\hline & Cardiology & 1583 & 16,852 & 9.4 \\
\hline & ICU & 973 & 2515 & 38.7 \\
\hline & Intervention & 772 & 3499 & 22.1 \\
\hline & Cardiac Surgery & 720 & 6208 & 11.6 \\
\hline \multirow[t]{5}{*}{2015} & Cardiology & 1664 & 18,535 & 9.0 \\
\hline & Psychiatry & 1445 & 3435 & 42.1 \\
\hline & $\begin{array}{l}\text { Endovascular } \\
\text { Surgery }\end{array}$ & 1331 & 3422 & 38.9 \\
\hline & ICU & 1040 & 2622 & 39.7 \\
\hline & Intervention & 1033 & 7097 & 14.6 \\
\hline \multirow[t]{5}{*}{2016} & Cardiology & 2721 & 21,169 & 12.9 \\
\hline & Psychiatry & 1655 & 3496 & 47.3 \\
\hline & $\begin{array}{l}\text { Endovascular } \\
\text { Surgery }\end{array}$ & 978 & 4346 & 22.5 \\
\hline & Cardiac Surgery & 901 & 4696 & 19.2 \\
\hline & Intervention & 864 & 8372 & 10.3 \\
\hline \multirow[t]{5}{*}{2017} & Cardiology & 3165 & 26,274 & 12.0 \\
\hline & Psychiatry & $|78|$ & 3677 & 48.4 \\
\hline & ICU & 1079 & 4242 & 25.4 \\
\hline & $\begin{array}{l}\text { Endovascular } \\
\text { Surgery }\end{array}$ & 951 & 5294 & 18.0 \\
\hline & Cardiac Surgery & 919 & 3692 & 24.9 \\
\hline \multirow[t]{5}{*}{2018} & Cardiology & 2940 & 30,299 & 9.7 \\
\hline & Intervention & 1937 & 6718 & 28.8 \\
\hline & Psychiatry & 1873 & 4041 & 46.3 \\
\hline & Cardiac Surgery & 1010 & 6526 & 15.5 \\
\hline & ICU & 1005 & 4600 & 21.8 \\
\hline
\end{tabular}

to genotyping of CYP2C19 (50.2\%) and MTHFR (41.7\%), and other genotyping of pharmacogenes such as CYP2C9, $V K O R C 1$ and $A L D H$ constituted only a small proportion. In fact, over 40 drug-gene pairs could be genotyped in our hospital. However, other PGx testing besides the items in the present study were too few to be negligible and the other PGx testing used a different genotyping platform and could not be checked in PACS system, therefore we excluded their data in the study. Importantly, due to the very low frequency of variant alleles in the Chinese population, physicians encounter very few variant genotypes of some drug genes. This undermines their confidence in the validity of the PGx test and their eagerness for the next new test to open. Indeed, These observations warrant further study to confirm the perceptions of Chinese physicians' perceptions of PGx.
Pharmacists could play an important role in the clinical implementation of PGx tests in the primary care. ${ }^{16}$ However, the majority of more than 300 pharmacists in our hospital had no or little knowledge about the pharmacogenomics because of the absence of PGx education in their major during the master or doctor school year. Interpretation and reports of PGx testing results were bear by the pharmacists accounting for less than $10 \%$ of all the pharmacist in the hospital, over two-thirds of whom work in the pharmacy. Except providing the genotypes and their functional effect, the reports could not be more personalized considering the other factors of the genotyped patients such as age, sex, disease state and combined drugs and so on. These would add to the already heavy workload of the physicians when they interpret the reports of the PGx testing, knowledge of which was lacking in physicians like in the pharmacists. Therefore, continuing education of pharmacogenomics in the physicians and pharmacists would be necessary measures to take in the future for a better adoption of PGx test in primary care. ${ }^{30}$

A better individualized health-care model delivering the PGx testing results in time and integrating an available informatics support had been proved to be imperative in the clinical implementation of PGx testing. ${ }^{31}$ The PGx testing results were integrated into the PACS system among CT, MRI, and so on, which have not been fully integrated into the electronic drug prescribing and dispensing systems, and we found some PGx tests genotyping the same variant were repeatedly performed by two different departments through different physicians (data not shown). This might result from the inconvenience of getting all the PGx testing results once in a check, on the contrary obtaining which only one by one. All the PGx testing results should be integrated into one single pharmacogenomics information page, instead of scattered independently within other imaging and pathology results.

Our present study demonstrated that few departments performed the majority of PGx testing and decreased number of departments performing the PGx tests were also observed. Prescription of clopidogrel in cardiology, neurology, and cardiac surgery and folate usage in psychiatry might explain the majority of CYP2C19 and MTHFR genotyping in these departments.

In contrast to the growing adoption of PGx tests in these departments, those departments occasionally performed PGx testing gradually abandoned them after considering the imbalance between the burden of opening and interpreting the PGx information and their effectiveness in the clinical decisionmaking of physicians. This less-optimistic trend suggested a shrinkage of clinical implementation of PGx in a wide health- 
care range, which might cause severe clinical sequences when considering the stop of promotion of scarce PGx testing such as $H L A-B * 58: 01$ and allopurinol, $H L A-B * 15: 02$ and carbamazepine. ${ }^{32,33}$ Development of a panel of actionable variants of pharmacogenes for PGx testing like PGx-Passport could be a robust tool to resolve the above mentioned problems. ${ }^{4,34}$ However, these tools should be localized considering the special genetic variation profiling of pharmacogenes in special ethnic populations. Importantly, a total of 200 million Chinese minorities had been under-representative in the pharmacogenomics studies such as Uyghur Chinese with an admixture between Caucasians and East Asians. ${ }^{35}$ Economic development of China promoted the migration of many minorities into the interior areas from far remote Western China, which increased the encounter of the minority Chinese by the physicians lacking of knowledge about the PGx data in the ethnic Chinese populations. Further studies and clinical implementation of PGx could provide a more precise medication to Han Chinese and other minority Chinese.

Decreased cost of new genotyping techniques such as next-generation sequencing (NGS) have made these cutting-edge methods more feasible and available in the clinical implementation of pharmacogenomics NGS could find more rare (with a variant frequency less than 0.01 ) variants of pharmacogenes in the resequenced individual and the imperative role these rare variants playing in the drug responses such as warfarin, simvastatin, voriconazole, olanzapine, and irinotecan had been identified. ${ }^{36-38}$ In addition, other long-reads single molecule real-time (SMRT) sequencing technologies such as Oxford Nanopore Technologies (ONT) or SMRT Pacific Biosciences (PacBio) sequencing platforms could resolved the complex pharmacogene genomic loci such as $C Y P 2 D$ including CYP2D8P, CYP2D7, and CYP2D6, which was annoying puzzle in the NGS with short-reads. ${ }^{39}$ Altogether, these advanced sequencing techniques could provide the clinicians a very accurate variants spectrum of an individual patient, plus the bioinformatics aid, to realize the precise medication.

\section{Conclusion}

In summary, a total of 47,265 PGx testing results were obtained with an average annual rate of growth of $63.0 \%$ across five years. A $50.2 \%$ of all the PGx testing results were for the determination of $C Y P 2 C 19 * 2$, *3 genotypes, and $41.7 \%$ were for the MTHFR C677T genotype. The main top five departments were cardiology, psychiatry, ICU, cardiac surgery and intervention, respectively. Clinical implementation of PGx testing in China is growing rapidly, but the types and implementing departments of PGx testing were limited. Our present study reported the real-world implementation modality of PGx tests in China. It will help us to understand the testing of PGx in China in order to promote the rational development of PGx.

\section{Acknowledgments}

This study was supported by the Young Investigators Foundation of The First Affiliated Hospital of Zhengzhou University (YNQN2015192 and YNQN2017125).

\section{Disclosure}

The authors declare that they have no conflicts of interest in this work.

\section{References}

1. Relling M, Evans W. Pharmacogenomics in the clinic. Nature. 2015;526(7573):343-350. doi:10.1038/nature 15817

2. Cecchin E, Stocco G. Pharmacogenomics and personalized medicine. Genes. 2020;11(6):679. doi:10.3390/genes11060679

3. Marin JJG, Serrano MA, Monte MJ, et al. Role of genetic variations in the hepatic handling of drugs. Int J Mol Sci. 2020;21(8):2884. doi:10.3390/ijms21082884

4. van der Wouden $\mathrm{CH}$, van Rhenen MH, Jama W, et al. Development of the PGx-passport: a panel of actionable germline genetic variants for pre-emptive pharmacogenetic testing. Clin Pharmacol Ther. 2019;106(4):866-873. doi:10.1002/cpt.1489

5. Simon T, Verstuyft C, Mary-Krause M, et al. Genetic determinants of response to clopidogrel and cardiovascular events. $N$ Engl $J$ Med. 2009;360(4):363-375. doi:10.1056/NEJMoa0808227

6. Paré G, Mehta SR, Yusuf S, et al. Effects of CYP2C19 genotype on outcomes of clopidogrel treatment. $N$ Engl J Med. 2010;363 (18):1704-1714. doi:10.1056/NEJMoa. 1008410

7. Martin J, Williams AK, Klein MD, et al. Frequency and clinical outcomes of CYP2C19 genotype-guided escalation and de-escalation of antiplatelet therapy in a real-world clinical setting. Genet Med. 2020;22(1):160-169. doi:10.1038/s41436-019-0611-1

8. Frigon MP, Mè B, Dubois-Bouchard C, Gagnon AL, Tardif S, Tremblay K. Pharmacogenetic testing in primary care practice: opinions of physicians, pharmacists and patients. Pharmacogenomics. 2019;20(8):589-598. doi:10.2217/pgs-2019-0004

9. Albassam A, Alshammari S, Ouda G, Koshy S, Awad A. Knowledge, perceptions and confidence of physicians and pharmacists towards pharmacogenetics practice in Kuwait. PLoS One. 2018;13(9): e0203033. doi:10.1371/journal.pone.0203033

10. Keeling NJ, Rosenthal MM, West-Strum D, Patel AS, Haidar CE, Hoffman JM. Preemptive pharmacogenetic testing: exploring the knowledge and perspectives of US payers. Genet Med. 2019;21 (5):1224-1232. doi:10.1038/gim.2017.181

11. Wagner M, Jasek M, Karabon L. Immune checkpoint molecules-inherited variations as markers for cancer risk. Front Immunol. 2021;11:606721. doi:10.3389/fimmu.2020.606721

12. Brunham LR, Baker S, Mammen A, Mancini GBJ, Rosenson RS. Role of genetics in the prediction of statin-associated muscle symptoms and optimization of statin use and adherence. Cardiovasc Res. 2018;114(8):1073-1081. doi:10.1093/cvr/cvy119 
13. van der Wouden CH, Swen JJ, Samwald M, Mitropoulou C, Schwab M, Guchelaar HJ. A brighter future for the implementation of pharmacogenomic testing. Eur J Hum Genet. 2016;24 (12):1658-1660. doi:10.1038/ejhg.2016.116

14. Sperber NR, Carpenter JS, Cavallari LH, et al. Challenges and strategies for implementing genomic services in diverse settings: experiences from the Implementing GeNomics In pracTicE (IGNITE) network. BMC Med Genomics. 2017;10(1):35. doi:10.1186/s12920-017-0273-2

15. Shuldiner AR, Relling MV, Peterson JF, et al. The pharmacogenomics research network translational pharmacogenetics program: overcoming challenges of real-world implementation. Clin Pharmacol Ther. 2013;94(2):207-210. doi:10.1038/clpt.2013.59

16. Bank P, Swen JJ, Schaap RD, Klootwijk DB, Baak-Pablo R, Guchelaar HJ. A pilot study of the implementation of pharmacogenomic pharmacist initiated pre-emptive testing in primary care. Eur J Hum Genet. 2019;27(10):1532-1541. doi:10.1038/s41431-019-0454-x

17. Johnson JA, Caudle KE, Gong L, et al. Clinical pharmacogenetics implementation consortium (CPIC) guideline for pharmacogenetics-guided warfarin dosing: 2017 update. Clin Pharmacol Ther. 2017;102(3):397-404. doi:10.1002/cpt.668

18. Scott SA, Sangkuhl K, Stein CM, et al. Clinical pharmacogenetics implementation consortium guidelines for CYP2C19 genotype and clopidogrel therapy: 2013 update. Clin Pharmacol Ther. 2013;94 (3):317-323. doi:10.1038/clpt.2013.105

19. Lima JJ, Thomas CD, Barbarino J, et al. Clinical Pharmacogenetics Implementation Consortium (CPIC) Guideline for CYP2C19 and proton pump inhibitor dosing. Clin Pharmacol Ther. 2021;109 (6): 1417-1423. doi:10.1002/cpt.2015

20. Moriyama B, Obeng AO, Barbarino J, et al. Clinical Pharmacogenetics Implementation Consortium (CPIC) guideline for CYP2C19 and voriconazole therapy. Clin Pharmacol Ther. 2017;102 (1):45-51. doi:10.1002/cpt.583

21. Hicks JK, Bishop JR, Sangkuhl K, et al. Clinical Pharmacogenetics Implementation Consortium (CPIC) guideline for CYP2D6 and CYP2C19 genotypes and dosing of selective serotonin reuptake inhibitors. Clin Pharmacol Ther. 2015;98(2):127-134. doi:10.1002/ cpt.147

22. Cao Y, Xu J, Zhang Z, et al. Association study between methylenetetrahydrofolate reductase polymorphisms and unexplained recurrent pregnancy loss: a meta-analysis. Gene. 2013;514(2):105-111. doi:10.1016/j.gene.2012.10.091

23. Wu W, Shen O, Qin Y, et al. Methylenetetrahydrofolate reductase C677T polymorphism and the risk of male infertility: a meta-analysis. Int J Androl. 2012;35(1):18-24. doi:10.1111/j.13652605.2011.01147.x

24. Chen H, Yang X, Lu M. Methylenetetrahydrofolate reductase gene polymorphisms and recurrent pregnancy loss in China: a systematic review and meta-analysis. Arch Gynecol Obstet. 2016;293 (2):283-290. doi:10.1007/s00404-015-3894-8

25. Du B, Shi X, Yin C, Feng X. Polymorphisms of methalenetetrahydrofolate reductase in recurrent pregnancy loss: an overview of systematic reviews and meta-analyses. J Assist Reprod Genet. 2019;36(7):1315-1328. doi:10.1007/s10815-019-01473-2

Pharmacogenomics and Personalized Medicine

\section{Publish your work in this journal}

Pharmacogenomics and Personalized Medicine is an international, peer-reviewed, open access journal characterizing the influence of genotype on pharmacology leading to the development of personalized treatment programs and individualized drug selection for improved safety, efficacy and sustainability. This journal is indexed
26. Wan L, Li Y, Zhang Z, Sun Z, He Y, Li R. Methylenetetrahydrofolate reductase and psychiatric diseases. Transl Psychiatry. 2018;8(1):242. doi:10.1038/s41398-018-0276-6

27. Hua Y, Zhao H, Kong Y, Ye M. Association between the MTHFR gene and Alzheimer's disease: a meta-analysis. Int $J$ Neurosci. 2011;121(8):462-471. doi:10.3109/00207454.2011.578778

28. Hu CY, Qian ZZ, Gong FF, et al. Methylenetetrahydrofolate reductase (MTHFR) polymorphism susceptibility to schizophrenia and bipolar disorder: an updated meta-analysis. $J$ Neural Transm. 2014;122(2):307-320. doi:10.1007/s00702-014-1261-8

29. Professional committee on rational clinical use of drugs, China Association of Pharmaceutical Education. Multidisciplinary expert consensus on rational clinical folic acid supplementation in china. Chin J Front Med Sci. 2020;12(11):19-36. doi:10.12037/YXQY.2020.11-05

30. Mills R, Haga SB. Qualitative user evaluation of a revised pharmacogenetic educational toolkit. Pharmgenomics Pers Med. 2018;11:139-146. doi:10.2147/PGPM.S169648

31. O’Donnell PH, Bush A, Spitz J, et al. The 1200 patients project: creating a new medical model system for clinical implementation of pharmacogenomics. Clin Pharmacol Ther. 2012;92(4):446-449. doi:10.1038/clpt.2012.117

32. Hung SI, Chung WH, Liou LB, et al. HLA-B*5801 allele as a genetic marker for severe cutaneous adverse reactions caused by allopurinol. Proc Natl Acad Sci U S A. 2005;102(11):4134-4139. doi:10.1073/ pnas.0409500102

33. Fang H, Xu X, Kaur K, et al. A screening test for HLA-B*15:02 in a large United States patient cohort identifies broader risk of carbamazepine-induced adverse events. Front Pharmacol. 2019;10:149. doi:10.3389/fphar.2019.00149

34. Bank P, Swen JJ, Guchelaar HJ. Estimated nationwide impact of implementing a preemptive pharmacogenetic panel approach to guide drug prescribing in primary care in the Netherlands. $B M C$ Med. 2019;17(1):110. doi:10.1186/s12916-019-1342-5

35. Qi G, Li D, Zhang X. Genetic variation of cytochrome P450 in Uyghur Chinese population. Drug Metab Pharmacokinet. 2018;33 (1):55-60. doi:10.1016/j.dmpk.2017.02.002

36. Giannopoulou E, Katsila T, Mitropoulou C, Tsermpini EE, Patrinos GP. Integrating next-generation sequencing in the clinical pharmacogenomics workflow. Front Pharmacol. 2019;10:384. doi:10.3389/fphar.2019.00384

37. Ng D, Hong CS, Singh LN, Johnston JJ, Mullikin JC, Biesecker LG. Assessing the capability of massively parallel sequencing for opportunistic pharmacogenetic screening. Genet Med. 2017;19(3):357-361. doi:10.1038/gim.2016.105

38. Ingelman-Sundberg M, Mkrtchian S, Zhou Y, Lauschke VM. Integrating rare genetic variants into pharmacogenetic drug response predictions. Hum Genomics. 2018;12(1):26. doi:10.1186/s40246-0180157-3

39. Laver TW, Caswell RC, Moore KA, et al. Pitfalls of haplotype phasing from amplicon-based long-read sequencing. Sci Rep. 2016;6(1):21746. doi:10.1038/srep21746

on the American Chemical Society's Chemical Abstracts Service (CAS). The manuscript management system is completely online and includes a very quick and fair peer-review system, which is all easy to use. Visit http://www.dovepress.com/testimonials.php to read real quotes from published authors. 